\title{
O ENSINO DA CANCEROLOGIA NA ENFERMAGEM NO BRASIL E A CONTRIBUIÇÃO DA ESCOLA PAULISTA DE ENFERMAGEM- UNIVERSIDADE FEDERAL DE SÃO PAULO
}

\author{
Maria Gaby Rivero de Gutiérrez'1, Edvane Birelo Lopes De Domenico², Marléa Chagas Moreira3, \\ Lúcia Marta Giunta da Silva ${ }^{4}$
}

\begin{abstract}
${ }^{1}$ Doutora em Enfermagem. Professor Associado da Escola Paulista de Enfermagem da Universidade Federal de São Paulo (UNIFESP). São Paulo, Brasil. E-mail: gaby.gutierrez@unifesp.br

${ }^{2}$ Doutora em Enfermagem. Professor Adjunto da UNIFESP. São Paulo, Brasil. E-mail: domenico.edvane@unifesp.br

${ }^{3}$ Doutora em Enfermagem. Professor Adjunto da Escola de Enfermagem Anna Nery da Universidade Federal do Rio de Janeiro. Rio de Janeiro, Brasil. E-mail: marleachagas@gmail.com

${ }^{4}$ Doutoranda do Programa de Pós-Graduação em Enfermagem da UNIFESP. São Paulo, Brasil. E-mail: luciagiunta@terra,com.br
\end{abstract}

\begin{abstract}
RESUMO: O controle do câncer e a melhoria do atendimento requerem investimentos de educação. Objetivamos neste artigo, atualizar a retrospectiva histórica para implantação do ensino da cancerologia nos cursos de graduação em enfermagem no Brasil e descrever os avanços no ensino dessa temática na Escola Paulista de Enfermagem da Universidade Federal de São Paulo. Estudo descritivo, de perspectiva histórica, com análise documental. Fontes primárias: relatórios dos eventos. Fontes secundárias: artigos de enfermagem publicados em periódicos nacionais. O caráter dinâmico e coletivo da construção da proposta de ensino da oncologia foi decisivo para sua estruturação paradigmática e operacional à luz das concepções de transversalidade e interdisciplinaridade; pela defesa da articulação entre instituições formadoras e prestadoras de serviço, e pela política de atualização profissional contínua. Concluímos que os avanços não foram homogêneos nas diversas regiões do país e nas instituições de ensino e de saúde, indicando a necessidade de retomada do processo.
\end{abstract}

DESCRITORES: Educação em enfermagem. Enfermagem oncológica. Artigo histórico.

\section{TEACHING MEDICAL ONCOLOGY IN NURSING IN BRAZIL AND THE CONTRIBUTION FROM ESCOLA PAULISTA DE ENFERMAGEM AT THE FEDERAL UNIVERSITY OF SÃO PAULO}

\begin{abstract}
Cancer control and improvement in health care services require investment in education. We aim in this paper to update the historical retrospective in order to implement medical oncology teaching in nursing undergraduate courses in Brazil and to describe the teaching advancements in this subject at the Paulista Nursing School at the Federal University of São Paulo, Brazil (Escola Paulista de Enfermagem da Universidade Federal de São Paulo). This is a descriptive study with a historical perspective, using documental analysis. Primary sources were event reports. Secondary sources were nursing articles published in national periodicals. The dynamic and collective character of creating the teaching proposal for medical oncology was decisive in developing its pragmatic and operational structure under the light shed by the concepts of cross-section and interdisciplinary aspects; and by the defense of articulation between educational and service institutions, and by the policy of continuous professional improvement. We conclude that advancements were not homogeneous in the different Brazilian regions and among teaching and health institutions; indicating the need to resume the process.
\end{abstract}

DESCRIPTORS: Education, nursing. Oncology nursing. Historical article.

\section{LA ENSEÑANZA DE LA CANCEROLOGÍA EN LA ENFERMERÍA BRASILEÑA Y LA CONTRIBUCIÓN DE LA ESCUELA PAULISTA DE ENFERMERÍA DE LA UNIVERSIDAD FEDERAL DE SÃO PAULO}

RESUMEN: Para el control del cáncer y la mejora de la atención se requiere de inversión en educación. El objetivo de este estudio es actualizar la retrospectiva histórica de la implantación de la enseñanza de la cancerología en los cursos de pregrado en enfermería en Brasil y describir los avances en la enseñanza de esa temática en la Escuela Paulista de Enfermería de la Universidad Federal de São Paulo. Es un estudio descriptivo, de carácter histórico, con análisis documental. Fuentes primarias: informes de los eventos científicos. Fuentes secundarias: tesis y artículos de enfermería publicados en revistas nacionales. El carácter dinámico y colectivo de la construcción de la propuesta de enseñanza de la oncología fue decisivo para su estructuración paradigmática y su funcionamiento según conceptos interdisciplinarios y de transversalidad; como también, por la defensa de los vínculos entre instituciones de enseñanza y prestadoras de servicio, y por la política de actualización profesional continua. Se concluye que los avances no fueron homogéneos en las diferentes regiones del país ni en las instituciones de educación y salud, lo que indica la necesidad de retomada del proceso.

DESCRIPTORES: Educación en Enfermería. Enfermería oncológica. Artículo histórico. 


\section{INTRODUÇÃO}

Mantendo-se em $2^{\circ}$ lugar entre as causas de morte no país, cada vez mais confirmando as semelhanças nos padrões de incidência e mortalidade dos países desenvolvidos, o câncer no Brasil, também detém características evolutivas da doença demarcadas pela grande desinformação da população em geral e acesso precário as instituições especializadas. ${ }^{1}$

Essas características sentenciam a manutenção de diagnósticos em fases tardias de desenvolvimento da neoplasia acarretando, em muitas situações, estados permanentes de mutilações, perdas de capacidades orgânicas funcionais que levam a aposentadorias precoces, quando não a própria morte do indivíduo, num ciclo contínuo de sofrimento pessoal, desarranjos familiares emocionais e financeiros e comprometimento de recursos da área social da saúde e da economia do próprio país. ${ }^{2}$

Certamente o desenvolvimento econômico, avanço tecnológico, crescimento industrial, ingresso da mulher no mercado de trabalho, envelhecimento populacional, são fatores que, desprovidos de condições educacionais capazes de gerarem na população consciência dos fatores de risco relacionados ao câncer, bem como, do direito de usufruir dos exames diagnósticos e tratamentos comprovadamente eficientes, tornam-se ingredientes para a condição desfavorável no controle do avanço do câncer em nossa sociedade. ${ }^{3-4}$

Muitas ações relacionadas ao controle do câncer dependem do nível de informação da população, desde os profissionais da saúde que devem ser capazes de prevenir, diagnosticar, tratar, evoluir e saber notificar os casos de câncer, até o cidadão morador da zona urbana ou rural. A amplitude da tarefa não é fácil de ser vencida e, por essa razão, sabe-se que há muito por avançar nesta área.

Assim, passados 10 anos da apresentação do trabalho Oncology teaching in the undergraduation nursing courses in Brazil, no $17^{\text {th }}$ International Cancer Congress, ${ }^{5}$ sentimos a necessidade de retomar o tema, visando analisá-lo à luz das demandas contemporâneas para a formação profissional de enfermeiros que possam atuar de forma efetiva na prevenção, detecção precoce, diagnóstico, tratamento, reabilitação e cuidados paliativos, de acordo com princípios da integralidade e humanização instituídos pela Política Nacional de Atenção Oncológica. Para tanto, tomaremos como base alguns dados contidos nesse documento para abordar, neste artigo, alguns movimentos nacionais que tiveram como foco a inserção do ensino da oncologia* nos cursos de graduação em enfermagem.

\section{OBJETIVOS}

Atualizar a retrospectiva histórica e as ações realizadas no processo de implantação do ensino da cancerologia nos cursos de graduação em enfermagem no Brasil.

Descrever os avanços no ensino da oncologia em enfermagem na Escola Paulista de Enfermagem (EPE) da Universidade Federal de São Paulo (UNIFESP).

\section{MÉTODO}

Estudo de perspectiva histórica, pois, “[...] fazer história como conhecimento e como vivência é recuperar a ação de diferentes grupos que nela atuam, procurando entender por que o processo tomou um dado rumo, e não o outro. Significa resgatar as injunções que permitiram a concretização de uma possibilidade, e não de outras" . ${ }^{6: 4}$

As fontes primárias utilizadas foram os relatórios dos eventos contendo as instituições engajadas, diretrizes e/ou recomendações. As fontes secundárias referem-se a artigos de enfermagem publicados em periódicos nacionais que focalizaram o assunto. Para a análise dos dados foi adotado o método da análise documental. ${ }^{7}$

\section{EDUCAÇÃO E ONCOLOGIA: PRIMEIROS MOVIMENTOS DE ESTRUTURAÇÃO}

Iniciaremos esta retrospectiva pelo ano de 1987, ocasião em que se realizou, em Brasília-DF, o I Simpósio Brasileiro de Educação em Cancerologia, coordenado pela então Divisão Nacional de Doenças Crônico-Degenerativas do Ministério da Saúde (MS), pois, a partir da temática abordada - O Ensino da Cancerologia nos Cursos de Graduação em Medicina, Odontologia e Enfermagem - surgiram diretrizes para a inclusão de conteúdos desta especialidade nos currículos dos referidos

\footnotetext{
Neste estudo, as palavras oncologia e cancerologia serão utilizadas como sinônimos, conforme consta do Descritores em Ciências da Saúde.
} 
cursos, no sentido de adequar a formação dos futuros profissionais às necessidades da população e dos serviços de saúde.

Como resultado desse encontro, e com base em levantamento realizado sobre a situação do ensino da cancerologia nos cursos de graduação em enfermagem, ${ }^{8}$ o grupo de estudos que representava a área de enfermagem propôs, entre outras ações, a criação de uma disciplina específica, que deveria contemplar a prevenção, detecção precoce e diagnóstico do câncer, incluindo, também, os aspectos de tratamento e reabilitação dos pacientes.

Em novembro do mesmo ano (1987), em Florianópolis-SC, ocorreu o I Congresso Brasileiro de Enfermagem Oncológica, cuja recomendação relativa à educação em Cancerologia, em nível de graduação, foi a de implantar conteúdos curriculares de Enfermagem Oncológica, reforçando, desta forma, a proposta anterior.

O movimento subsequente aconteceu em dezembro de 1987, quando pela primeira vez, reuniu-se a Comissão Nacional para o Ensino da Cancerologia nos Cursos de Graduação em Enfermagem, que havia sido formalizada pelo Programa de Oncologia (Pro-Onco), da então Campanha Nacional de Combate ao Câncer (CNCC) do MS. Esta Comissão foi composta por enfermeiros docentes e assistenciais, oriundos das cinco macrorregiões brasileiras e, entre outros temas, foram discutidas as diretrizes para o ensino dessa temática e definidas as competências requeridas para o enfermeiro na área da cancerologia. ${ }^{9}$

Os avanços obtidos nas reuniões e eventos de 1988 foram decisivos para a estruturação paradigmática e operacional do ensino da oncologia à luz das concepções de transversalidade e interdisciplinaridade. Defendeu-se que os conteúdos relativos ao câncer em seus diferentes aspectos perpassassem as disciplinas que compunham as matrizes curriculares da época e que, além da integração dos saberes e convergência nos objetivos educacionais, houvesse movimentos de articulação entre instituições formadoras e prestadoras de serviço, como também uma política contínua de atualização dos conhecimentos e experiências.

O documento que trazia essas propostas foi aprovado em fevereiro de 1988, e cópias foram enviadas às Escolas de Enfermagem do país, à Secretaria da Comissão de Especialistas do Ensino de Enfermagem do Ministério da Educação e Cultura e à Associação Brasileira de Enfermagem. ${ }^{9}$
Em que pese o esforço e compromisso das pessoas e instituições envolvidas nesse processo, fatores de diversas ordens impediram o alcance do impacto esperado. Assim, o Instituto Nacional do Câncer (INCA) e o Departamento de Enfermagem da UNIFESP firmaram, em 1990, um convênio de cooperação técnico-científica, sob a égide da integração institucional e do trabalho coletivo. Acordou-se também, o acompanhamento e a avaliação contínua das atividades e etapas a serem colocadas em prática para viabilizar o desenvolvimento desse processo.

Deste convênio várias iniciativas foram implementadas, entre as quais se destaca a realização, em 1992 e 1995, na cidade de São Paulo, do I e II Seminário Nacional sobre o Ensino da Cancerologia nos Cursos de Graduação em Enfermagem.

\section{SEMINÁRIOS NACIONAIS SOBRE O EN- SINO DA CANCEROLOGIA NOS CURSOS DE GRADUAÇÃO EM ENFERMAGEM}

No I Seminário, as discussões tiveram por base as informações obtidas de 60 escolas $(63,8 \%)$ do universo das 96 em funcionamento em junho de $1992 .{ }^{10}$ A situação analisada não mostrou mudanças substanciais em relação aos levantamentos realizados por outros pesquisadores. ${ }^{7,11}$

Tendo em vista que a finalidade do evento foi debater estratégias para operacionalizar a inserção de conteúdos de enfermagem em oncologia nos cursos de graduação, as discussões ocorridas sobre as propostas elaboradas no documento de 1988, foram fundamentais para sensibilizar os participantes, envolvendo-os como sujeitos no processo de mudança.

Entre as recomendações, destaca-se a de que a referida proposta fosse discutida com o corpo docente e discente das escolas e, quando possível, com os enfermeiros dos campos de prática, com vistas a reorientar os programas já existentes ou implantá-los nas escolas em que esses conteúdos não fossem ministrados.

As 96 escolas de enfermagem do Brasil existente em junho de 1992 receberam esse Programa, que apontava os fundamentos dos conteúdos da cancerologia, tendo por eixos a situação epidemiológica do câncer e o ensino de Enfermagem, o qual inclui, também, as bases gerais para o preparo geral do enfermeiro em cancerologia abrangendo as competências, o conteúdo pro- 
gramático e sua distribuição na grade curricular, métodos de ensino, avaliação do alunado e do programa educativo. ${ }^{12}$

Outros compromissos assumidos nesse evento foram: ${ }^{* *}$

- elaboração de um livro de texto para fundamentar o conteúdo desse programa de ensino, cuja produção foi coordenada pelo INCA, editado em 1995, como resultado de um esforço multiprofissional e multi-institucional, encontrando-se na sua terceira edição, revisada e ampliada, que está disponível na íntegra e gratuitamente no site do INCA; ${ }^{13}$

- implementação de um processo de monitoramento da aderência das Escolas de Enfermagem ao programa proposto. Para tanto, foram realizados estudos, um nacional ${ }^{14}$ e cinco regionais. ${ }^{15-19}$ Os de âmbito regional subsidiaram as discussões do II Seminário Nacional sobre o Ensino da Cancerologia nos Cursos de Graduação em Enfermagem, em 1995.

A apresentação do processo de implementação do Programa de Ensino da Cancerologia propiciou a análise das dificuldades regionais e foram aprovadas propostas e estratégias de ação, com vistas a se superar os pontos críticos identificados e possibilitar o avanço da implementação do mesmo. ${ }^{20}$

Considerando as diferenças das realidades existentes nas macrorregiões, foi aprovada a idéia de realização de eventos regionais para adequar o programa de ensino às reais necessidades locais e estaduais. Fato este ocorrido nos seminários realizados na Região Sul (Londrina-PR) em 1996, e outro na Região Sudeste, na Escola de Enfermagem Anna Nery da Universidade Federal do Rio de Janeiro, em 1997. Em ambos houve a participação do INCA, na organização e realização dos mesmos em parceria com instituições de ensino que sediaram os eventos.

Um dos aspectos a destacar no seminário da região sudeste, constante em seu documento oficial, foi a preocupação com o planejamento das atividades do evento, direcionado-as para o fazer da enfermagem em oncologia, isto é, "para as experiências vivenciadas em cada estado, com vistas a uma reflexão sobre o tema", ${ }^{21: 10}$ dentre as quais, as estratégias que vem sendo utilizadas para minimizar as dificuldades encontradas pelas escolas para a implantação do programa de ensino da cancerologia nos cursos de graduação em enfermagem.

Em suma, os resultados obtidos no período compreendido entre 1992 e 1997, evidenciaram, por um lado, avanços significativos e, por outro, a persistência de algumas dificuldades, aspectos estes, relatados sucintamente a seguir:

- a mobilização de professores, alunos e enfermeiros assistenciais para discutir e buscar formas de operacionalizar a implantação do Programa de Ensino da Cancerologia;

- o poder de pressão exercido pelos acadêmicos para que mudanças ocorressem, tanto no ensino como na utilização de serviços de saúde para a prática da assistência de enfermagem em cancerologia;

- desenvolvimento, ainda que incipiente, da integração interdisciplinar face à natureza multiprofissional desse programa de ensino;

- ampliação das experiências de aprendizagem pela inclusão de campos de estágio que oferecem, além da assistência curativa, atividades de prevenção e detecção precoce do câncer,

- prestação de serviços voltados para a reabilitação e cuidados paliativos, em instituições especializadas, ou não, com extensão ao domicílio e que, paralelamente, desenvolvessem atividades de preparo de cuidadores;

- emergência e consolidação de alguns grupos de estudo a partir de necessidades sentidas, quer da clientela-alvo, quer da formação de recursos humanos, visando a melhoria da qualificação para se atuar de forma mais efetiva nessa área.

\section{PARTICIPAÇÃO DOS GRUPOS DE ESTU- DO, PESQUISA E SOCIEDADE DE ESPE- CIALISTAS EM ONCOLOGIA}

Os grupos de estudo e pesquisa, constituídos principalmente por enfermeiros docentes e assistenciais, surgiram em instituições de ensino que desenvolviam programas de pós-graduação, propiciando o delineamento de linhas de pesquisa, cujos projetos aglutinavam estudantes de graduação e pós-graduação em defesa da expansão da Enfermagem em Oncologia quanti e qualitativamente.

Os projetos de pesquisa fecundados e nascidos desses grupos estavam, e ainda estão vinculados às áreas assistencial, de formação de recursos

** Os documentos de 1988, 1992 e 1995, podem ser obtidos na íntegra no site do Núcleo de Enfermagem em Oncologia (NEO) (http://www.unifesp.br/denf/neo), no menu de opções Educação, na pasta Documentos Históricos. 
humanos e de organização de serviços de saúde e de enfermagem, visando à promoção de mudanças na qualificação profissional e na prestação de cuidados integrais à clientela-alvo da oncologia.

Outro espaço de defesa e promoção da área é a Sociedade Brasileira de Enfermagem Oncológica (SBEO), criada em 1988 e que, em 1996, inseriu-se ao lado de outras sociedades de especialistas na Revista Brasileira de Cancerologia, patrocinada pelo INCA, tem promovido a veiculação de trabalhos produzidos por enfermeiros nesta área de conhecimento, ampliando assim as fontes de divulgação em periódicos credenciados junto à comunidade acadêmica.

\section{Trajetória de 1997 aos dias atuais}

O que há de investigação na área após as análises e propostas de 1997, decorre de pesquisa, que teve como um dos objetivos atualizar os dados relativos ao processo de implementação do ensino da cancerologia nos cursos de graduação em enfermagem. ${ }^{22}$

Os resultados reiteraram diagnósticos anteriores, como o ensino restrito a algumas aulas avulsas e experiências práticas esporádicas e a escassez ou falta de conteúdos teóricos e práticos sobre reabilitação e cuidados paliativos. Entretanto, alguns avanços foram identificados por essa investigação, entre os quais a inclusão de experiências práticas em ações de promoção, prevenção e detecção precoce do câncer e o interesse de docentes para participar de cursos de capacitação em enfermagem em oncologia.

Considerando, que a investigação descrita foi divulgada em 2008, torna-se evidente que ocorreu o distanciamento das instituições envolvidas no processo, permanecendo os vínculos pessoais e de proximidade geográfica. Uma das possibilidades analíticas sobre essa condição pode ser creditada ao fato de os profissionais mais envolvidos com a idealização e operacionalização do movimento, embora representassem instituições, encontraremse em diferentes fases da trajetória profissional, como: em condição de aposentadoria, necessitando dedicar-se ao ensino da pós-graduação, de se titularem para atendimento de exigência na carreira acadêmica, entre outras possíveis razões. Ou seja, não houve a continuidade necessária para que esses movimentos tivessem prosseguimento.

Também cabe evidenciar a falta da repercussão normativa para as mudanças almejadas nas instituições de classe, como Conselho Federal de Enfermagem/Conselho Regional de Enfermagem, na própria sociedade de especialistas, SBEO e nas representações de enfermagem em âmbito da regulamentação do ensino superior/Ministério da Educação, responsáveis pela construção das diretrizes curriculares divulgadas anos após.

\section{AVANÇOS DO ENSINO DA ONCOLOGIA EM DIFERENTES NÍVEIS DE FORMAÇÃO DA EPE/UNIFESP}

A exemplo do que acontece em diferentes instituições de ensino, ${ }^{9}$ uma das estratégias que vem sendo desenvolvida e ampliada na EPE/ UNIFESP, é o investimento efetivo na capacitação dos presentes e futuros profissionais de saúde, para atuar de forma mais incisiva na prevenção, detecção precoce, diagnóstico e tratamento do câncer, assim como na reabilitação do doente.

A base de sustentação das atividades didático-pedagógicas e investigativas se dá pelo modelo explicitado na Figura 1.

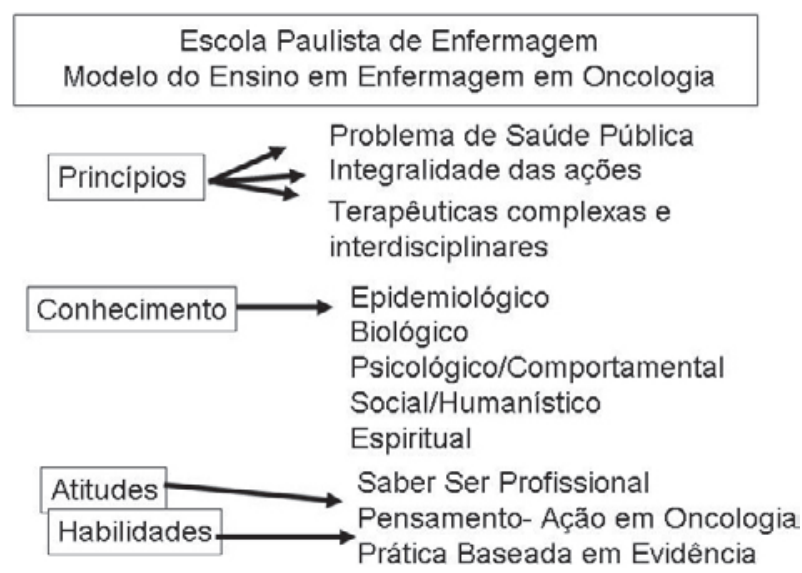

Figura 1 - Modelo de ensino de Enfermagem em Oncologia no Curso de Graduação da EPE/ UNIFESP. São Paulo - SP, 2009

Nesse modelo, as propostas oriundas dos seminários nacionais e regionais, bem como dos seminários realizados na EPE/UNIFESP (1993 e 1996) estão incluídas e atualizadas de acordo com as demandas curriculares atuais e tendências, como a prática baseada em evidências.

Em relação à distribuição dos conteúdos referentes à oncologia geral e à enfermagem em oncologia, estes estão distribuídos ao longo das quatro séries do curso, nas diferentes disciplinas que compõem a matriz curricular vigente. Ressalta-se que há uma preocupação em integrar os conteúdos 
teórico-práticos curriculares com as atividades de extensão e pesquisa, incentivando os estudantes a participarem do projeto de extensão, Acolhe-Onco, e do Núcleo de Enfermagem Oncológica (NEO).

Como forma de divulgar os conhecimentos em enfermagem em oncologia e, primordialmente, criar um espaço interativo, dinâmico, atual e atraente aos estudantes, profissionais, pacientes/ familiares/cuidadores e população em geral, o NEO criou um site (www.unifesp.br/denf/neo), em novembro de 2008. A Figura 2 representa a proposta de transversalidade e opções de atividades presentes na capacitação de graduandos na área de oncologia.

Conteúdos de oncologia em graduação

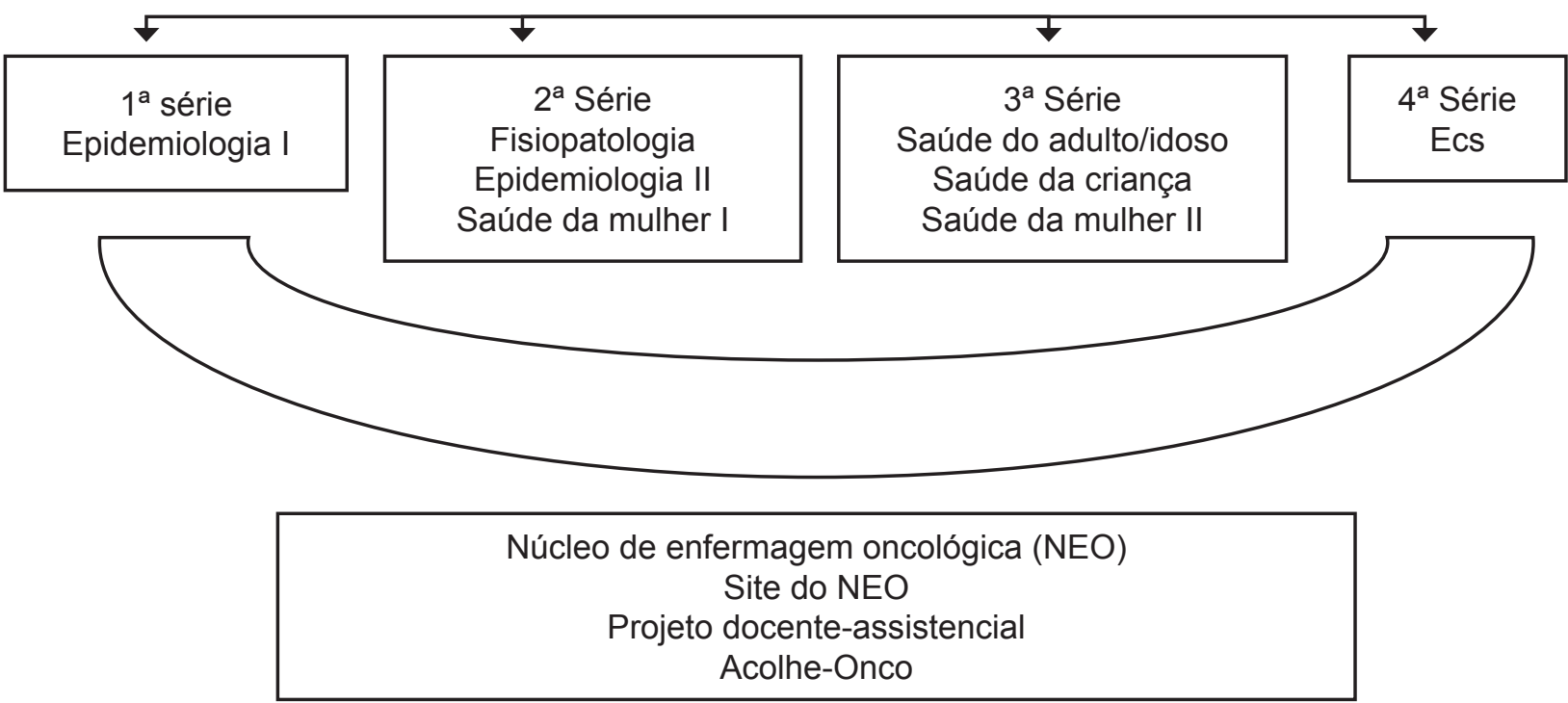

Figura 2 - Distribuição dos conteúdos teórico-práticos da Enfermagem em Oncologia no Curso de Graduação da EPE/UNIFESP. São Paulo - SP, 2009

Essa descrição é fruto da reformulação desses conteúdos no currículo da graduação, ainda em processo de consolidação e o que pode ser observado, na prática, é um interesse crescente dos alunos sobre a temática do câncer. Entretanto, também já se pode analisar que a conformação curricular, com o tempo de permanência do estudante na instituição de ensino quase que totalmente ocupado com as atividades curriculares obrigatórias, limita a possibilidade de participação e desenvolvimento de projetos vinculados ao desejo individual de construção de competências profissionais específicas, no caso, competências e habilidades na enfermagem em oncologia.

\section{CONCLUSÃO}

Reconhece-se que, no transcurso de duas décadas (1988-2009) de discussão da problemática em foco, os avanços não são homogêneos, tanto nas diversas regiões do país, quanto nas instituições de ensino e de saúde, face às desigualdades sócio-econômicas existentes que propiciam a concentração de profissionais qualificados, bem como de recursos materiais e tecnológicos nas áreas mais desenvolvidas.

Para finalizar a presente atualização do processo de inserção de conteúdos de oncologia na graduação, apresentamos situações que precisam ser devidamente discutidas e colocadas em escala de prioridade por uma nova proposta de aliança entre os enfermeiros dos diferentes espaços de atuação, SBEO, INCA e demais instâncias normativas e de referência na oncologia brasileira. Os pontos sugeridos são: garantir o exercício da especialidade, assegurar a qualidade da assistência, promover intercâmbios entre os especialistas 
enfermeiros e equipe multiprofissional, incentivar e aprimorar as pesquisas, retomar o movimento de consolidação dos conteúdos de oncologia em diferentes níveis de formação superior, incentivar a participação de enfermeiros especialistas na SBEO e construir diretrizes capazes de nortear o planejamento da Educação Permanente da Enfermagem em Oncologia.

\section{REFERÊNCIAS}

1. Ministério da Saúde (BR), Secretaria Nacional de Assistência à Saúde, Instituto Nacional de Câncer, Coordenação de Prevenção e Vigilância. Atlas de mortalidade por câncer no Brasil 1996-2009 [online]. Rio de Janeiro (RJ): INCA; 2008 [acesso 2009 Fev 25]. Disponível em: http:/ / mortalidade.inca.gov.br/

2. Borges DML, Sena MF de, Ferreira MAF, Roncalli AG. Mortalidade por câncer de boca e condição sócioeconômica no Brasil. Cad Saúde Pública [online]. 2009 Fev [acesso 2009 Fev 25]; 25(2):321-7. Disponível em: http://www.scielo.br/scielo.php?script=sci arttext\&pid=S0102-311X2009000200010\&lng=es

3. Zago A, Pereira LAA, Braga ALF, Bousquat A. Mortalidade por câncer de mama em mulheres na Baixada Santista, 1980 a 1999. Rev Saúde Pública [online]. 2005 Aug [acesso 2009 Fev 25] 39(4):641-5. Disponível em: http:/ / www.scielo. br / scielo.php?script=sci_arttext\&pid=S003489102005000400019\&lng=en

4. Mendoza-Sassi R, Béria JU. Prevalence of having a regular doctor, associated factors, and the effect on health services utilization: a populationbased study in Southern Brazil. Cad Saúde Pública [online]. 2003 Out [acesso 2009 Fev 25]; 19(5):1257-66. Disponível em: http:/ / www.scielosp. org/scielo.php?script=sci_arttext\&pid=S0102311X2003000500004\&lng=en

5. Gutiérrez, MGR, Adami NP, Castro RAP, Maranhão AMS, Lavor MF, Gadelha MIP. Oncology Teaching In The Undergraduation Nursing Courses In Brazil [abstract]. In: Proceedings of the 17th International Cancer Congress; 1998 Ago 24-8; Rio de Janeiro. Rio de Janeiro (RJ): UICC/INCA RJ; 1998. p.198.

6. Vieira MPDA, Peixoto MRC, Khoury YMA. A pesquisa em história. $4^{\mathrm{a}}$ ed. São Paulo (SP): Ática; 1998.

7. Cometto MC, Piovan M, Gomez P. Aportes de los coloquios panamericanos a la investigación en enfermería: período 2000-2006. Texto Contexto Enferm [online]. 2008 Out-Dez [acesso 2009 Fev 25]; 17(4):720-6. Disponível em: http://www. scielo.br/scielo.php?script=sci_arttext\&pid=S010407072008000400013\&lng=pt\&nrm=iso\&tlng=es

8. Rodrigues C, Queiroz I. A situação atual do ensino de Enfermagem Oncológica nos cursos de graduação em enfermagem do Brasil. Rev Paul Enf. 1988 JanJun; 8(1):23-5.
9. Ministério da Saúde (BR). Campanha Nacional de Combate ao Câncer/Sistema Integrado e Regionalizado de Controle do Câncer. Ensino da Cancerologia nos cursos de graduação em Enfermagem. Rio de Janeiro (RJ): MS: 1988.

10. Gutiérrez MGR, Castro RAP, Aguinaga S. O ensino da cancerologia nos cursos de graduação em enfermagem: por que e para quê? Rev Bras Cancerol. 1993 Jan-Mar; 39(1):11-20.

11. Cezareti IUR, Gutiérrez MGR, Sabates AL, Erhart ERN, Pereira AL. Estudo sobre o ensino da oncologia nas Escolas de Enfermagem da Grande São Paulo. Acta Paul Enf. 1991 Jan-Mar; 4(1):5-10.

12. Ministério da Saúde (BR), Instituto Nacional de Câncer, Coordenação de Programas de Controle do Câncer. Ensino da cancerologia nos cursos de graduação em Enfermagem [online]. Rio de Janeiro (RJ): Escola Paulista de Medicina. Departamento de Enfermagem; 1992 [acesso 2009 Fev 15]. Disponível em: http://www.unifesp.br/denf/ neo/index.php?option $=$ com_phocadownload\& view $=$ category \&id=1:docshist\&download=2:ens ino-da-cancerologia-nos-cursos-de-graduao-emenfermagem-1992\&Itemid=74

13. Ministério da Saúde (BR). Instituto Nacional de Câncer. Ações de enfermagem para o controle do cáncer: uma proposta de intergação ensino-serviço [online]. $3^{\mathrm{a}}$ ed. Rio de Janeiro (RJ): INCA; 2008 [acesso 2009 Fev 15]. Disponível em: http:/ / www. inca.gov.br/enfermagem/index.asp

14. Vianna LAC, Schirmer J. Avaliação da aderência à proposta de ensino em Cancerologia na graduação pelas Escolas de enfermagem do Brasil. Rev Bras Cancerol. 1994 Out-Dez 40(4):215-17.

15. Riker LL, Gomes VM. O processo de implantação do programa de ensino da Cancerologia nos cursos de graduação em Enfermagem da Região Norte. In: Anais do $2^{\circ}$ Seminário Nacional sobre o Ensino da Cancerologia nos Cursos de Graduação em Enfermagem; 1995 Jun 8-10; São Paulo. São Paulo (SP): INCA/Dep.Enf.-UNIFESP; 1995 [acesso 2009 Fev 17]. Disponível em: http://www.unifesp.br/ denf/neo/index.php?option=com_phocadownloa $\mathrm{d} \&$ view $=$ category \&id $=1$ :docshist \&download $=3: p$ rocesso-de-implantao-do-programa-de-ensino-dacancerologia-nos-cursos-de-graduao-em-enfermagem\&Itemid $=74$

16. Lima WR. O ensino da cancerologia nos cursos de graduação em enfermagem da Região Nordeste. In: Anais do $2^{\circ}$ Seminário Nacional sobre o Ensino da Cancerologia nos Cursos de Graduação em Enfermagem; 1995 Jun 8-10; São Paulo. São Paulo (SP): INCA/Dep.Enf.-UNIFESP; 1995 [acesso 2009 Fev 17]. Disponível em: http://www.unifesp.br/ denf/neo/index.php?option=com_phocadownlo ad\&view = category \&id=1: docshist\&download =6: o-ensino-da-cancerologia-nos-cursos-dde-graduaoem-enfermagem-na-regio-nordeste\&Itemid $=74$ 
17. Oliveira LMAC, Barbosa MA, Teles SA. O programa de ensino da cancerologia nas escolas de enfermagem da Região Centro-Oeste. In: Anais do $2^{\circ}$ Seminário Nacional sobre o Ensino da Cancerologia nos Cursos de Graduação em Enfermagem; 1995 Jun 8-10; São Paulo. São Paulo (SP): INCA/ Dep.Enf.-UNIFESP; 1995 [acesso 2009 Fev 17]. Disponível em: http://www.unifesp.br/denf/ neo/index.php?option $=$ com_phocadownload\& view $=$ category \&id $=1$ : docshist $\&$ download $=5: 0-$ programa-de-ensino-da-cancerologia-nas-escolasde-enfermagem-da-regio-centro-oeste\&Itemid $=74$

18. Gutiérrez MGR, Maranhão MAS, Adami NP, Castro RAP. Panorama dos processos de implantação/ implementação do ensino da cancerologia nos cursos de graduação em enfermagem da Região Sudeste. In: Anais do $2^{\circ}$ Seminário Nacional sobre o ensino da Cancerologia nos cursos de Graduação em Enfermagem; 1995 Jun 8-10; São Paulo. São Paulo (SP): INCA/Dep.Enf.-UNIFESP; 1995 [acesso 2009 Fev 17]. Disponível em: http://www.unifesp.br/ denf/neo/index.php?option=com_phocadownloa $\mathrm{d} \&$ view $=$ category\&id=1:docshist\&download=7:pa norama-dos-processos-de-implantao-implementaodo-programa-de-ensino-da-cancerologia-noscursos-de-graduao-em-enfermagem-da-regiosudeste\&Itemid $=74$

19. Nicola AL, Oliveira BRG, Scopel VMP. Pesquisa sobre o ensino da cancerologia nos cursos de graduação em enfermagem da Região Sul. In: Anais do $2^{\circ}$ Seminário Nacional sobre o Ensino da Cancerologia nos Cursos de Graduação em Enfermagem; 1995 Jun 8-10; São Paulo. São Paulo (SP): INCA/Dep.Enf.-UNIFESP; 1995 [acesso 2009 Fev 17]. Disponível em: http://www.unifesp.br/ denf/neo/index.php?option=com_phocadownload \&view $=$ category\&id=1:docshist\&download=4:pesq uisa-sobre-o-ensino-da-cancerologia-nos-cursos-degraduao-em-enfermagem-da-regio-sul\&Itemid $=74$

20. Gutiérrez MGR, Maranhäo AMSA, Castro, RAP, Adami NP. Núcleo de enfermagem em oncologia: experiência relacionada à assistência, ensino e pesquisa. Acta Paul Enf [online]. 1996 Jan-Abr [acesso 2009 Fev 15]; 9(1):92-7. Disponível em: http://www.unifesp.br/denf/acta/1996/9_1/ pdf/art10.pdf

21. Moreira MC. Sobre o tema do I Seminário da Região Sudeste - "Ensinar e Cuidar: Desafios e Perspectivas da Enfermagem em Cancerologia". In: Anais do $1^{\circ}$ Seminário da Região Sudeste. 1997 Set. Rio de Janeiro, Rio de Janeiro (RJ): Ministério da Saúde/INCA/Pro-Onco. Escola de Enfermagem Anna Nery/UFRJ; 1997 [acesso 2009 Out 20]. p.10. Disponível em: http://www.unifesp.br/denf/ neo/index.php?option $=$ com_phocadownload\&vi ew $=$ category $\& \mathrm{id}=1$ : docshist $\&$ download $=8$ :ensina r-e-cuidar-desafios-e-perspectivas-da-enfermagemem-cancerologia\&Itemid $=74$

22. Barbosa MBA. La enseñanza de la enfermería oncológica en las instituciones escolares del Estado de Rio de Janeiro [tese]. Guadalajara (ES): Universidad de Alaclá- Departamento de Didáctica; 2008. 